\title{
Perspectivas da aplicação das conotoxinas bloqueadoras de canais para cálcio dependentes de voltagem no trauma medular
}

\author{
Perspectives for the application of voltage-dependent calcium channels \\ blockers conotoxin in spinal cord injury
}

\author{
Karen Maciel de Oliveira ${ }^{\mathrm{I}^{*}}$ Mário Sérgio Lima Lavor ${ }^{\mathrm{I}}$ Fabíola Bono Fukushima $^{\mathrm{II}}$ \\ Fátima Caetano Caldeira ${ }^{I I I}$ Eliane Gonçalves Melo
}

\section{- REVISÃO BIBLIOGRÁFICA -}

RESUMO

\begin{abstract}
O bloqueio dos canais para cálcio dependentes de voltagem é uma estratégia importante no tratamento do trauma medular, pois previne o influxo exacerbado do cálcio que participa ativamente em processos neurodegenerativos agudos, resultando em neuroproteção com melhora das funções neurológica. Dentre esses bloqueadores, as toxinas de caramujos marinhos são peptídeos com adequada estabilidade estrutural, estudadas pelas ações específicas em canais iônicos e receptores que interferem diretamente na liberação de neurotransmissores e na neuromodulação dos neurônios motores e sensitivos da medula espinal. Elas já são utilizadas no tratamento de desordens neurológicas e mostram-se promissoras no desenvolvimento de novas terapias para o trauma medular. Portanto, objetivou-se discorrer sobre a fisiopatologia do trauma medular e a possível utilização terapêutica das toxinas de caramujo marinho, atuantes nos principais canais para cálcio dependentes de voltagem.
\end{abstract}

Palavras-chave: conotoxinas, canais para cálcio, Conus magus, Conus geographus, trauma medular.

\section{ABSTRACT}

Blocking voltage dependent calcium channels is an important strategy in acute spinal trauma treatment, because it prevents the exacerbated calcium influx which participates actively in acute neurodegenerative processes, resulting in neuroprotection with improvement of neurological and electrophysiological functions. The cone snail toxins are peptides with adequate structural stability, which have been studied by specific actions on ion channels and receptors that directly interfering in the release of neurotransmitters and neuromodulation of sensory and motor neurons of the spinal cord. They are already used in the treatment of neurological disorders and appear to be promising in the development of new therapies for spinal trauma. Therefore, it was aimed to discuss the pathophysiology of spinal cord trauma, and possible therapeutic use of marine snail toxins that acts in voltagedependent calcium channels.

Key words: conotoxin, calcium channels, Conus magus, Conus geographus, spinal cord injury.

\section{INTRODUÇÃO}

O trauma medular espinal (TME) tem se tornado cada vez mais frequente devido ao aumento da violência urbana, sendo os acidentes automobilísticos, quedas e ferimentos por armas de fogo as causas mais comuns (EDDLESTON et al., 2000; LEE et al., 2013). As lesões medulares, devido a sua gravidade e irreversibilidade, geram transtorno emocional pela baixa qualidade de vida e transtorno socioeconômico pelos custos médicos, programas de reabilitação prolongada ou invalidez (ROSSIGNOL et al., 2007).

Na medicina veterinária, a incidência do trauma medular em cães ainda é desconhecida, mas foi estimado que cerca de $2 \%$ dos casos resultam da hérnia de disco, sendo mais frequente em raças como Dachshund (20-25\%) (BUBENIK \& HOSGOOD, 2008) e, mais comumente localizada na região toracolombar (52\%), devido ao estreitamento do canal vertebral nesta região (ZARDO et al., 2010; MENDES \& ARIAS, 2012). As principais causas incluem fatores extrínsecos, como atropelamentos

\footnotetext{
'Departamento de Medicina e Cirurgia Veterinárias, Universidade Federal de Minas Gerais (UFMG), Campus Pampulha, Av. Antônio Carlos, 6627, 30123-970, Belo Horizonte, MG, Brasil. E-mail: kamoliveira@yahoo.com.br. *Autor para correspondência.

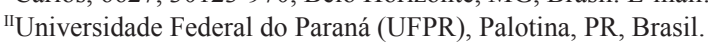

IIIUniversidade Estadual do Oeste do Paraná (UNIOESTE), Guarapuava, PR, Brasil. Recebido 16.07.12 Aprovado 18.09.13 Devolvido pelo autor 10.12.13 CR-2012-0538.R1
} 
$(66 \%)$, quedas $(7 \%)$ e brigas $(9 \%)$ (FIGHERA et al., 2008; MENDES \& ARIAS, 2012) e lesões intrínsecas, decorrentes de extrusão e protrusão do disco intervertebral, fraturas patológicas, anormalidades congênitas e instabilidade de vértebras (SEVERO et al., 2007; MENDES \& ARIAS, 2012).

Independente da causa do TME os déficits neurológicos, decorrentes da interrupção direta das vias neuronais (SEVERO et al., 2007), comprometem as funções motoras, sensitivas e autonômicas, implicando em perda parcial ou total dos movimentos voluntários e/ou da sensibilidade em membros torácicos e/ ou pélvicos (OLBY et al., 2003; BUBENIK \& HOSGOOD, 2008; MENDES \& ARIAS, 2012). Entre outras complicações, destacam-se também alterações no funcionamento dos sistemas urinário, intestinal, respiratório, circulatório e reprodutivo (ROSSIGNOL et al., 2007; FAILLI et al., 2012). A recuperação neurológica dependerá da gravidade, localização da lesão e tempo decorrido até o início do tratamento (CARLSON \& GORDEN, 2002).

Apesar de recentes avanços no estudo da fisiopatologia do TME (CAO \& DONG, 2013), ainda não foi estabelecido um tratamento efetivo que detenha a expansão da lesão secundária e diminua as sequelas permanentes (KWON et al., 2004). Clinicamente, há um grande interesse na utilização de novas terapias, como células tronco (DASARI et al., 2007), ou os bloqueadores de canais iônicos (OLIVEIRA, 2012; LAVOR, 2013), capazes de prevenir ou retardar as complicações neurológicas e, com isso, ampliar a janela terapêutica para outros potenciais agentes neuroprotetores. Dentre os diferentes tratamentos propostos, estão os antiinflamatórios esteroidais (BARTHOLDI \& SCHWAB, 1995; ROSADO, 2011) e não esteroidais (GUTH et al., 2006), bloqueadores de canais para sódio (SCHWARTZ \& FEHLINGS, 2001; WU et al., 2013), bloqueadores de canais para cálcio (BCC) (OLIVEIRA, 2012) e anestésicos gerais (FUKUSHIMA, 2012; LAVOR, 2013). Agentes BCC mostram-se especialmente promissores na redução de lesões cerebrais e medulares, por impedir o influxo exacerbado desse íon e, assim, prevenir a progressão da lesão secundária (VERWEIJ et al., 1997).

$\mathrm{Na}$ natureza, há uma extensa variedade de BCC a ser investigada, dentre eles, destacamse as toxinas de caramujos marinhos do gênero Conus, também denominadas conotoxinas. Sua estabilidade estrutural, facilidade de síntese, elevada especificidade de alvo e pequeno tamanho de seus peptídeos, proporcionam-lhes um importante potencial terapêutico (SHEN et al., 2000; LEWIS \& GARCIA, 2003; OLIVERA \& TEICHERT, 2007).
Assim, esta revisão objetiva discorrer sobre a fisiopatologia do TME, com ênfase na importância do cálcio $\left(\mathrm{Ca}^{2+}\right)$ no mecanismo de morte neuronal, abordando a possível utilização terapêutica das toxinas de caramujo marinho bloqueadoras de canais para $\mathrm{Ca}^{2+}$ dependentes de voltagem (CCDV).

Fisiopatologia do trauma medular

A lesão

A fisiopatologia do TME é caracterizada por um processo contínuo de destruição tecidual, reparação e cicatrização ao redor e no local da lesão. $\mathrm{O}$ impacto inicial no momento do trauma acarreta dano mecânico imediato da medula espinal (ME) e tecido adjacente, com ruptura de vasos sanguíneos, corpos neuronais, axônios e células da glia, resultando na interrupção fisiológica e/ou morfológica dos impulsos nervosos e na ativação de uma cascata de eventos destrutivos (SEVERO et al., 2007; OYINBO, 2011; JIA et al., 2013).

As lesões secundárias iniciam-se minutos após o trauma mecânico. A quebra da barreira hematoespinal desencadeia aumento da permeabilidade vascular, com formação de edema, início da resposta inflamatória, perda de cargas aniônicas e extravasamento de proteínas plasmáticas (ROWLAND et al., 2008). Concomitantemente, a ruptura da microvasculatura gera hemorragia que, associada ao edema, leva à isquemia medular, estendendo-se rostral e caudalmente(HULSEBOSCH, 2002; ROWLAND et al., 2008). Além disso, células gliais e endoteliais liberam substâncias vasoativas que se concentram próximo à lesão, contribuindo para a isquemia neuronal (GRILL, 2005). Ressalta-se que astrócitos localizados na periferia da lesão tornam-se reativos, proliferam e hipertrofiam, na tentativa de conter uma resposta inflamatória exacerbada, todavia pode dificultar a neuroplasticidade pela formação dessa cicatriz local (HERMANN et al., 2008).

\section{O papel do cálcio no trauma medular}

Paralelamente a esses eventos, ocorrem outros processos que intensificam a lesão neuronal, tendo destaque a excitotoxicidade. Ela causa desequilíbrio eletrolítico significativo (KWON et al., 2004; SEVERO et al., 2007), resultante da liberação excessiva e recaptação inadequada do glutamato (LEPORE et al., 2011), com consequente transmissão sináptica excitatória prolongada (HULSEBOSCH, 2002; ROWLAND et al., 2008). Isso acarreta o influxo acentuado de sódio e $\mathrm{Ca}^{2+}$ pela ativação de receptores ionotrópicos e metabotrópicos, além da entrada do $\mathrm{Ca}^{2+}$ pelos $\mathrm{CCDV}$, abertos com a despolarização da membrana (BERRIDGE, 2002). 
A ativação de CCDV constitui uma das vias mais importantes de entrada de $\mathrm{Ca}^{2+} \mathrm{e}$ representa um passo chave na regulação dos processos celulares (UCHITEL, 1997) e da excitabilidade celular, que participam ativamente em processos neurodegenerativos agudos (AGRAWAL et al., 2000). Os CCDV são divididos em duas grandes classes fisiológicas, baseadas nas voltagens de ativação (voltagem de baixo limiar - tipo T ou alto limiar - tipo L, N, P/Q e R) e três subfamílias, designadas como tipo Cav. 1 (L), Cav. 2 (N, P/Q e R) e a Cav.3 (T), com base na similaridade de suas características farmacológicas. $\mathrm{Na} \mathrm{ME}$, foi evidenciada a participação dos CCDV tipo $\mathrm{N}, \mathrm{P} / \mathrm{Q}$ e $\mathrm{R}$ na corrente de $\mathrm{Ca}^{2+}$ dos motoneurônios (CARLIN et al., 2000).

Com o influxo exagerado de cálcio, a mitocôndria torna-se um alvo importante, pois, na tentativa de interromper os potenciais eventos letais, ela sequestra esse íon (SANTO-DOMINGO E DEMAUREX, 2010). Contudo, o excesso de cálcio mitocondrial contribui para ativar a cascata de apoptose com geração de espécies reativas de oxigênio, edema mitocondrial e liberação de fatores apoptogênicos, como citocromo $c$, fator indutor de apoptose, entre outros, que ativam caspases executoras, como caspase- 3 e caspase-7 (Figura 1) (BERRIDGE, 2002; SULLIVAN et al., 2004; DECUYPERE et al., 2011). A apoptose é um mecanismo essencial durante o desenvolvimento do sistema nervoso central para eliminação de células excessivas. Entretanto, após o TME, tem-se mostrado como fator importante na perda celular, sendo detectada de horas a semanas após a lesão, em diversos tipos celulares, como oligodendrócitos, neurônios, neutrófilos, microglia e macrófagos (LIU et al., 1996; HALL \& SPRINGER, 2004).

Gravidade e recuperação neurológica

A lesão inicial, portanto, se expande progressivamente dentro de poucas horas, causando perdas de segmentos funcionais ainda maiores, com características de desmielinização parcial ou total, responsável pelos déficits de condução (GRILL, 2005; SEVERO et al., 2007). Se a substância branca remanescente possuir mais porções intactas dos

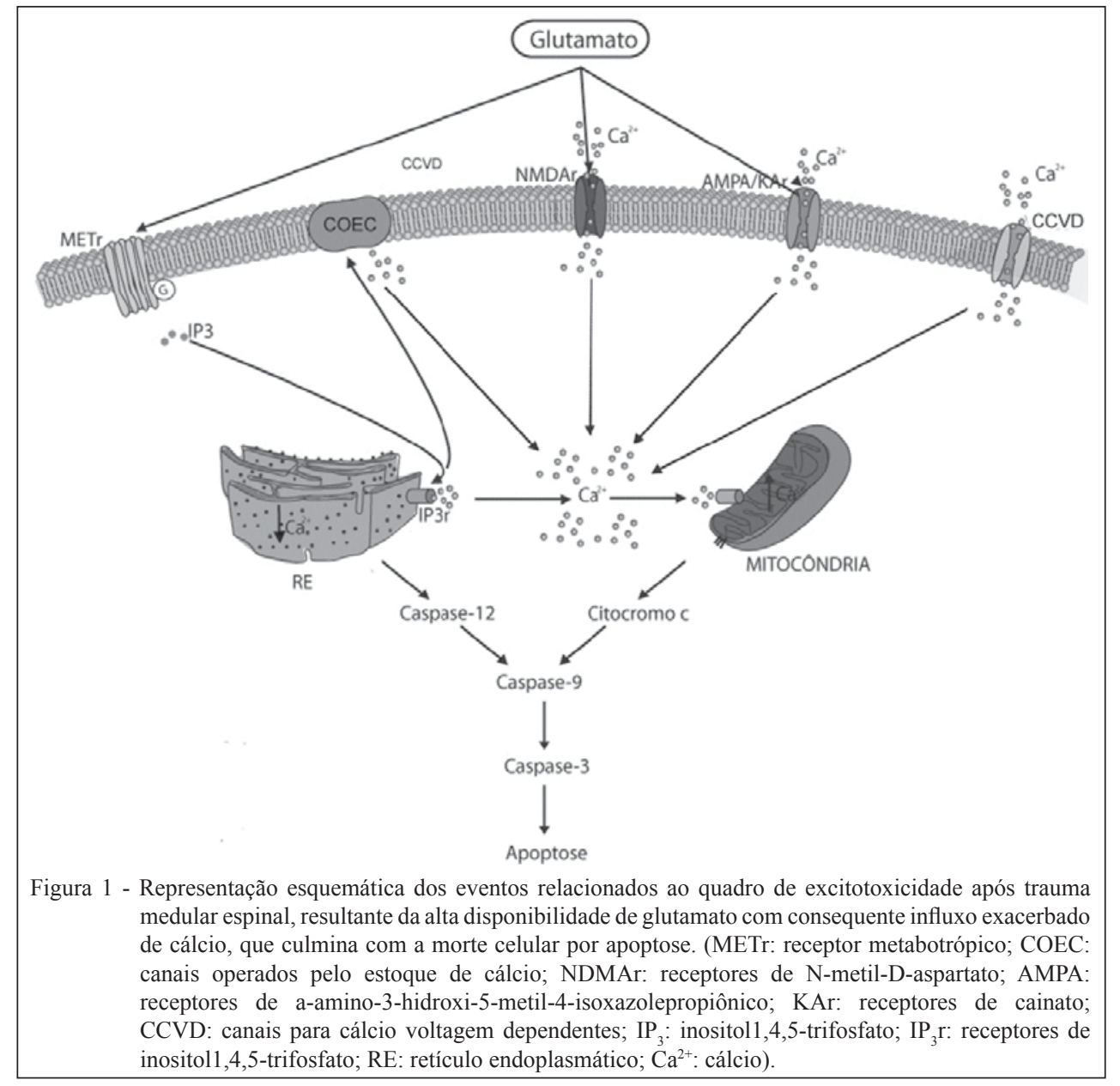

Ciência Rural, v.44, n.3, mar, 2014. 
tratos ascendentes sensitivos e descendentes motores, maiores serão as chances da recuperação funcional (HALL \& SPRINGER, 2004). Pesquisas mostraram que BCC são agentes farmacológicos promissores na redução de lesões cerebrais (VERWEIJ et al., 1997; BERMAN et al., 2000; ASLAN et al., 2009) e medulares (OLIVEIRA, 2012; LAVOR, 2013), ao impedir o influxo exacerbado desse íon e, dessa forma, prevenir a progressão da lesão secundária.

Os bloqueadores de canais para cálcio dependentes de voltagem

O bloqueio de CCDV é uma estratégia neuroprotetora importante, por estar envolvido na cascata de eventos deletérios pós-trauma (IMAIZUMI et al., 1999; LAVOR, 2013). No TME, alguns estudos evidenciaram que o bloqueio de CCDV tipo $\mathrm{N}$ e L tem papel fundamental em reduzir lesões secundárias, resultando em neuroproteção com melhora das funções neurológica e eletrofisiológica (IMAIZUMI et al., 1999; AGRAWAL et al., 2000). Pesquisas realizadas por nosso grupo corroboram tais resultados. Ao utilizar modelo de isquemia medular espinal in vitro, demonstrou-se que os bloqueadores de CCDV tipo $\mathrm{N}$ e $\mathrm{P} / \mathrm{Q}$ reduziram a morte neuronal e a liberação de glutamato e de lactato desidrogenase (LDH) no corno ventral da medula (LAVOR, 2013). Especula-se, dessa forma, que o bloqueio dos CCDV proteja a integridade estrutural dos oligodendrócitos e astrócitos, devido à presença de canais do tipo $\mathrm{N}$ em suas membranas, envolvidos no controle de diversos neurotransmissores e polipeptídeos que medeiam à resposta nociceptiva, propiciando uma melhor preservação na condução axonal após o TME (AGRAWAL et al., 2000).

Nos canais para $\mathrm{Ca}^{2+}$, a subunidade $\alpha-1$ é o principal alvo dos bloqueadores. Os bloqueadores inorgânicos incluem íons divalentes e trivalentes, como níquel e cádmio, utilizados como ferramentas de pesquisas, mas pouco práticos para uso clínico. Os bloqueadores orgânicos como diidropiridina, fenilalquilaminas e benzodiazepinas, bloqueadores dos canais do tipo L, prescritos para doenças cardiovasculares e enxaquecas, participam pouco na liberação de neurotransmissores (DIAZ \& DICKENSON, 1997). Asubfamília de canais para $\mathrm{Ca}^{2+}$, condutores de correntes do tipo $\mathrm{N}$ e $\mathrm{P} / \mathrm{Q}$, é insensível aos clássicos antagonistas, mas são bloqueados seletivamente por peptídeos de toxinas isolados de aranhas e caramujos marinhos (CAMERINO et al., 2007). Desde a descoberta da interação de toxinas com os CCDV, seu uso nas afecções neurológicas tem sido investigado (OLIVERA et al., 1987).
Toxinas de caramujos marinhos

Pesquisadores, frente às características terapêuticas já observadas, persistem na investigação das conotoxinas à procura de moléculas biologicamente ativas, como peptídeos neuroativos e neuroprotetores, derivados de diferentes espécies venenosas que possam ser aplicados clinicamente (SHEN et al., 2000).

Há quase 30 anos, o primeiro componente ativo do veneno de caramujo marinho do gênero Conus foi caracterizado bioquimicamente, instigado pelo modo como esses animais paralisavam as presas. Eles são predadores de águas tropicais e subtropicais, com aproximadamente 500 espécies, classificadas em três grupos, de acordo com o hábito alimentar: vermívoros, moluscívoros e piscívoros (SHEN et al., 2000). As espécies de caramujos marinhos com alvos já estabelecidos incluem C. magus, C. aulicus, C. regius, $C$. purpurascens, $C$. imperialis e $C$. geographus (OLIVERA \& TEICHERT, 2007). O veneno do caramujo marinho do gênero Conus causa acidente grave, com quadros de dor intensa, seguida de parestesia generalizada, além da paralisia neuromuscular e óbito por insuficiência respiratória relatados em humanos (WATTERS \& STOMMEL, 2004).

Essas toxinas têm sido estudadas por ter ações específicas em canais iônicos e em receptores para neurotransmissores (KAPOOR, 2010). Para sua obtenção, ela é extraída dos ductos de veneno desses animais e purificada por fracionamento, pela técnica de cromatografia líquida de alta eficiência em fase reversa, baseada em superfície de óxidos inorgânicos (AGUILAR et al., 2009). A toxina purificada é composta por pequenos peptídeos, denominados conopeptídeos, variando o tamanho de oito a 31 aminoácidos, enriquecida com pontos de dissulfeto (SHEN et al., 2000), os quais mostraram resultados promissores em estudos experimentais de desordens neurológicas, como epilepsia (MALMBERG et al., 2003), Parkinson, Alzheimer, isquemia medular (LAVOR, 2013) e já são utilizados em síndromes neuropáticas de dores crônicas (LIVETT et al., 2004; MEHDIRATTA \& SABERWAL, 2007).

Atualmente, conhece-se cerca de 10 superfamílias de conotoxinas com alta seletividade para um alvo molecular específico como canais iônicos ou receptores (OLIVERA \& CRUZ, 2001). Elas são subdivididas em diversas famílias ou classes. As ômega-conotoxinas ( $\omega$-conotoxinas) são específicas para CCDV, inibindo a liberação de neurotransmissores. Outras famílias bloqueiam receptores nicotínicos (alfa- e psi-conotoxina), canais para potássio voltagem dependentes (mu- 
e delta-conotoxina), receptores de serotonina (sigma-conotoxina) e inibição de canais iônicos inespecificamente (gama-conotoxina) (LIVETT et al., 2004; MEHDIRATTA \& SABERWAL, 2007; OLIVERA \& TEICHERT, 2007).

No TME, como visto anteriormente, ocorre morte neuronal, devido ao aumento do $\mathrm{Ca}^{2+}$ intracelular, que pode ser prevenido com BCC. As $\omega$-conotoxinas, provenientes de $\boldsymbol{C}$. magus e $\boldsymbol{C}$. geographus, mostraram-se valiosas na pesquisa para identificação dos subtipos de CCDV envolvidos em determinados processos celulares (HILLYARD et al., 1992; LAVOR, 2013). Com essas descobertas, tornaram-se alvos de terapias direcionadas contra dores crônicas, danos isquêmicos cerebrais e medulares, algumas formas de epilepsia e ataxia. Ao antagonizar CCDV, mostraram também efeito neuroprotetor em modelos de isquemia cerebral em coelhos e ratos (MINAMI et al., 2001; PINHEIRO et al., 2009), isquemia (LAVOR, 2013) e trauma medular em ratos (OLIVEIRA, 2012). Com o bloqueio desses canais, previne-se a liberação excessiva de neurotransmissores, incluindo aminoácidos excitatórios, como glutamato e dopamina (ROBICHAUD et al., 1994), atuando como protetores da hiperexcitabilidade e supressores da atividade epileptiforme (YAMADA et al., 1994) e mostrando potencial neuroprotetor no trauma medular (OLIVEIRA, 2012).

Dentro dessa classe, a MVIIA, proveniente do caramujo marinho Conus magus, conhecida como SNX-111 ou Ziconotide, é potente BCC tipo N e foi aprovada pela Food and Drug Administration como droga terapêutica pelas suas propriedades analgésicas, sendo utilizada para tratamento da dor crônica intensa e neuropática do câncer em humanos (OLIVERA et al., 1994; BOWERSOX \& LUTHER, 1998; BINGHAM et al., 2010). Além disso, possui propriedades neuroprotetoras, devido à inibição de neurotransmissores excitatórios secundário à isquemia (VALENTINO et al., 1993; GONÇAVES et al., 2011) e ao trauma cerebral em ratos (HOVDA et al., 1994; BOWERSOX \& LUTHER, 1998; BERMAN et al., 2000). Ainda não há relatos da aplicação dessa toxina no trauma medular, projeto em andamento por este grupo de pesquisa.

Nessa mesma família de conotoxinas, a MVIIC, também proveniente do Conus magus, é estudada por ser um bloqueador de amplo espectro para canais para $\mathrm{Ca}^{2+}$ do tipo $\mathrm{N}$ e $\mathrm{P} / \mathrm{Q}$ (HILLYARD et al., 1992; LIU et al., 1996; MCDONOUGH et al., 2002). Estudos em isquemia cerebral (LIU et al., 1996; MCDONOUGH et al., 2002; PINHEIRO et al.,
2009) e medular (IMAIZUMI et al., 1999; LAVOR, 2013) in vitro demonstraram que a MVIIC reduziu significativamente o influxo do $\mathrm{Ca}^{2+}$ através dos CCDV do tipo $\mathrm{N}$ e $\mathrm{P} / \mathrm{Q}$, diminuindo a liberação do glutamato. Por algum tempo, a MVIIC foi utilizada apenas como ferramenta de pesquisa, alegando-se alta letalidade aos mamíferos (BOWERSOX et al., 1994). Entretanto, baseado nos efeitos promissores dessa toxina no tratamento de diferentes patologias (LIU et al., 1996; IMAIZUMI et al., 1999; PINHEIRO et al., 2009), este grupo de pesquisa, em um trabalho inédito no trauma medular in vivo, verificou que a toxina não provocou efeitos colaterais, como também demonstrou maior preservação de corpos neuronais e melhor recuperação da capacidade motora (OLIVEIRA, 2012).

Já a $\omega$-conotoxina GVIA, proveniente do caramujo marinho Conus geographus (OLIVERA et al., 1984), é a mais utilizada como ferramenta na neurociência, por inibir irreversivelmente a transmissão sináptica ao bloquear os canais para $\mathrm{Ca}^{2+}$ do tipo $\mathrm{N}$ (FELDMAN et al., 1987; OLIVERA et al., 1994). Ela tem sido investigada quanto aos efeitos analgésicos e neuroprotetores na ME (NEBE et al., 1998), inibindo marcadamente a liberação excitotóxica de dopamina, com aplicação tópica sobre a ME, durante isquemia medular (ROBICHAUD et al., 1994).

Devido à importância do fluxo de $\mathrm{Ca}^{2+}$ para a homeostasia celular, o bloqueio de seus diferentes canais pode causar efeitos colaterais. A MVIIA, em altas concentrações, gera hipotensão e alterações cardiovasculares no momento da administração, além de tremor generalizado e déficit locomotor reversível, 60 minutos após aplicação intratecal, com retorno à normalidade em 24 horas em ratos (MALMBERG \& YAKSH, 1994; SCOTT et al., 2002; SOUZA et al., 2008). Já a MVIIC, em altas doses, superiores a 300pmol, pode causar fraqueza muscular, problemas de coordenação e andar em círculos em poucos minutos após a injeção intracerebroventricular. Doses inferiores a 100pmol da MVIIC são mais seguras e, eventualmente, ocorre paralisia flácida (DALMOLIN et al., 2011) e arqueamento dorsal (HILLYARD et al., 1992). Apesar de a dose ser um fator limitante para o uso das toxinas, são necessárias quantidades picomolares para que se obtenha o efeito de inibição dos CCDV (Souza et al., 2011).

\section{Perspectivas}

O trauma medular é uma emergência médica que desencadeia danos irreversíveis ao tecido neuronal, sendo o grande desafio da neurologia em todos os tempos. As estratégias neuroprotetoras, como 
prevenção de descargas repetitivas nas fibras nervosas pelo bloqueio dos canais para sódio, prevenção da liberação excessiva de neurotransmissores pelo bloqueio dos CCDV, prevenção do influxo massivo de glutamato ou qualquer combinação desses três mecanismos, têm sido propostas. Contudo, ainda não foi estabelecido um tratamento efetivo que detenha a expansão secundária e diminua as sequelas. Nesse contexto, as conotoxinas BCC apresentam um grande potencial no tratamento do trauma medular, uma vez que estudos demonstraram resultados positivos em diversos experimentos in vitro e, isoladamente, in vivo. Frente às propriedades promissoras dessas toxinas, verifica-se a necessidade de mais estudos experimentais que investiguem a aplicação desses peptídeos em modelos in vivo no trauma medular.

\section{REFERÊNCIAS}

AGRAWAL, S.K. et al. Role of L- and N-type calcium channels in the pathophysiology of traumatic spinal cord white matter injury. Journal of Neuroscience, v.99, n.1, p.179-188, 2000. Disponível em: <http://dx.doi.org/10.1016/S0306-4522(00)00165-2>. Acesso em: 11 mar. 2013. doi: 10.1016/S0306-4522(00)00165-2.

AGUILAR, M.B. et al. Structural characterization of five posttranslationally modified isomorphs of a novel putative $\delta$-conotoxin from the vermivorous snail Conus delessertii from the Mexican Caribbean Sea. Peptides, v.30, p.458-466, 2009. Disponível em: $<$ http://dx.doi.org/10.1016/j.peptides.2008.12. 005>. Acesso em: 11 mar. 2013. doi: 10.1016/j.peptides.2008.12.005.

BERMAN, R.F. et al. Neurobehavioral protection by the neuronal calcium channel blocker ziconotide in a model of traumatic diffuse brain injury in rats. Journal of Neurosurgery, v.93, p.821-828, 2000. Disponível em: <http://dx.doi.org/10.3171/ jns.2000.93.5.0821>. Acesso em: 19 mar. 2013. doi: 10.3171/ jns.2000.93.5.0821.

BERRIDGE, M.J. The endoplasmic reticulum: a multifunctional signaling organelle. Cell Calcium, v.32, n.5-6, p.235-249, 2002. Disponível em: <http://dx.doi.org/10.1016/S0143416002001823>. Acesso em: 19 mar. 2013. doi: 10.1016/S0143416002001823.

BINGHAM, J. et al. Drugs from slugs - Past, present and future perspectives of $\omega$-conotoxin research. Chemico-Biological, v.183, p.1-18, 2010. Disponível em: <http://dx.doi.org/10.1016/j. cbi.2009.09. 021>. Acesso em: 19 mar. 2013. doi: 10.1016/j. cbi.2009.09.021.

BOWERSOX, S.S. et al. Selective N-type neuronal voltagesensitive calcium channel blocker, SNX-111, produces spinal antinociception in rat models of acute, persistent, and neuropathic pain. Journal of Pharmacology and Experimental Therapeutics, v.279, p.1243-1249, 1996. Disponível em: <http:// jpet.aspetjournals.org/content/279/3/1243.long>. Acesso em: 19 mar. 2013. PMID: 8968347.

BOWERSOX, S.S.; LUTHER R. Pharmacotherapeutic potential of omega-conotoxin MVIIA (SNX-111), an N-type neuronal calcium channel blocker found in venom of Conus magus.
Toxicon, v.36, n.11, p.1651-1658, 1998. Disponível em: <http:// dx.doi.org/10.1016/S0041-0101(98)00158-5>. Acesso em: 19 mar. 2013. doi: 10.1016/S0041-0101(98)00158-5.

BUBENIK, L.; HOSGOOD, G. Disc herniation and urinary bladder dysfunction managed by manual expression, indwelling catheterization or intermittent catheterization. Veterinary Surgery, v.37, n.8, p.791-800, 2008. Disponível em: <http:// dx.doi.org/10.1111/j.1532-950X.2008.00452.x>. Acesso em: 19 mar. 2013. doi: 10.1111/j.1532-950X.2008.00452.x.

CAMERINO, D.C. et al. Ion channel pharmacology. Neurotherapeutics, v.4, p.184-198, 2007. Disponível em: <http:// dx.doi.org/10.1016/j.nurt.2007.01.013>. Acesso em: 19 mar. 2013. doi: 10.1016/j.nurt.2007.01.013.

CAO, H.Q.; DONG, E.D. An update on spinal cord injury research. Neuroscience Bulletin, v.29, n.1, p.94-102, 2013. Disponível em: $<\mathrm{http} / /$ dx.doi.org/10.1007/s12264-012-1277-8>. Acesso em: 19 mar. 2013. doi: 10.1007/s12264-012-1277-8.

CARLIN, K.P. et al. Characterization of calcium currents in functionally mature mouse spinal motoneurons. European Journal of Neuroscience, v.12, p.1624-34, 2000. Disponível em: $<\mathrm{http}$ :/ dx.doi.org/10.1046/j.1460-9568.2000.00050.x>. Acesso em: 19 mar. 2013. doi: 10.1046/j.1460-9568.2000.00050.x.

CARLSON, G.D.; GORDEN, C. Current developments in spinal cord injury research. Spine Journal, v.2, p.116-128, 2002. Disponível em: $<$ http://dx.doi.org/10.1016/S1529-9430(01)00029-8>. Acesso em: 19 mar. 2013. doi: 10.1016/S1529-9430(01)00029-8.

DALMOLIN, G.D. et al. Antinociceptive effect of Brazilian armed spider venom toxin Tx3-3 in animal models of neuropathic pain. Pain, v.152, p.2224-2332, 2011. Disponível em: <http://dx.doi. org/10.1016/j.pain.2011.04.015>. Acesso em: 19 mar. 2013. doi: 10.1016/j.pain.2011.04.015.

DASARI, V.R. et al. Axonal remyelination by cord blood stem cells after spinal cord injury. Journal of Neurotrauma, v.24, n.2, p.391410, 2007. Disponível em: <http://dx.doi.org/10.1089/neu.2006. 0142>. Acesso em: 19 mar. 2013. doi: 10.1089/neu.2006.0142.

DECUYPERE, J. et al. $\mathrm{IP}_{3}$ receptors, mitochondria, and $\mathrm{Ca}^{2+}$ signaling: implications for aging. Journal of Aging Research, v.2011p.1-20, 2011. Disponível em: <http://dx.doi. org/10.4061/2011/ 920178>. Acesso em: 19 mar. 2013. doi: $10.4061 / 2011 / 920178$

DIAZ, A.; DICKENSON, A.H. Blockade of spinal N- and P-type, but not L-type, calcium channels inhibits the excitability of rat dorsal horn neurons produced by subcutaneous formalin inflammation. Pain, v.69, p.93-100, 1997. Disponível em: < http:// dx.doi.org/10.1016/S0304-3959(96)03271-X>. Acesso em: 19 mar. 2013. doi: 10.1016/S0304-3959(96)03271-X.

EDDLESTON, J.M. et al. Survival, morbidity, and quality of life after discharge from intensive care. Critical Care Medicine, v.28, p.2293-2299, 2000. Disponível em: <http://dx.doi.org/10.1097/ 00003246-200007000-00018>. Acesso em: 19 mar. 2013. doi: 10.1097/00003246-200007000-00018.

FAILLI, V. et al. Functional neurological recovery after spinal cord injury is impaired in patients with infections. Brain, v.135, p.32383250, 2012. Disponível em: <http://dx.doi.org/10.1093/ brain/ aws267>. Acesso em: 19 mar. 2013. doi: 10.1093/brain/aws267. 
FELDMAN, D.H. et al. Omega Conus geographus toxin: a peptide that blocks calcium channels. FEBS Letters, v.214, p.295-300, 1987. Disponível em: <http://dx.doi.org/10.1016/00145793(87)80073-X>. Acesso em: 19 mar. 2013. doi: 10.1016/00145793(87)80073-X.

FIGHERA, R.A. et al. Aspectos patológicos de 155 casos fatais de cães atropelados por veículos automotivos. Ciência Rural, v.38, n.5, p.1375-1380, 2008. Disponível em: <http://dx.doi.org/ 10.1590/ S0103-84782008000500028>. Acesso em: 19 mar. 2013. doi: $10.1590 / \mathrm{S} 0103-84782008000500028$.

FUKUSHIMA, F.B. Avaliação do efeito neuroprotetor do propofol e etomidato em ratos submetidos ao trauma medular espinal. 2012. 85f. Tese (Doutorado em Ciência Animal) - Escola de Veterinária, Universidade Federal de Minas Gerais, MG.

GRILL, R.J. User-defined variables that affect outcome in spinal cord contusion/compression models. Experimental Neurology v.196, p.1-5, 2005. Disponível em: <http://dx.doi.org/10.1016/ j.expneurol.2005.08.005>. Acesso em: 19 mar. 2013. doi: 10.1016/j.expneurol.2005.08.005

GUTH, L. et al. The unique histopathological responses of the injured spinal cord. Annals of the New York Academy of Sciences, v.890, n.1, p.366-384, 2006. Disponível em: <http:// dx.doi.org/10.1111/ j.1749-6632.1999.tb08017.x>. Acesso em: 19 mar. 2013. doi: 10.1111/j.1749-6632.1999.tb08017.x.

HALL, E.D.; SPRINGER, J.E. Neuroprotection and acute spinal cord injury: a reappraisal. NeuroRx, v.1, p.80-100, 2004. Disponível em: $<$ http://dx.doi.org/10.1602/neurorx.1.1.80>. Acesso em: 19 mar. 2013. doi: 10.1602/neurorx.1.1.80.

HERMANN, J.E. et al. STAT3 is a critical regulator of astrogliosis and scar formation after spinal cord injury. Journal of Neuroscience, v.28, n.28, p.7231-7243, 2008. Disponível em: $<$ http://dx.doi.org/10.1523/JNEUROSCI.1709-08.2008>. Acesso em: 19 mar. 2013. doi: 10.1523/JNEUROSCI.1709-08.2008

HILLYARD, D.R. et al. A new Conus peptide ligand for mammalian presynaptic $\mathrm{Ca}^{2+}$ channels. Neuron, v.9, n.1, p.6977, 1992. Disponível em: <http://dx.doi.org/10.1016/08966273(92)90221-X>. Acesso em: 19 mar. 2013. doi: 10.1016/08966273(92)90221-X

HOVDA, D.A. et al. Administration of an omega-conopeptide one hour following traumatic brain injury reduces ${ }^{45} \mathrm{Calcium}$ accumulation. Acta Neurochirurgica, suppl.60, p.521-523, 1994. Disponível em: <http://dx.doi.org/10.1007/978-3-7091-9334-1_143>. Acesso em: 19 mar. 2013. doi: 10.1007/978-3-7091-9334-1_1433.

HULSEBOSCH, C.E. Recent advances in pathophysiology and treatment of spinal cord injury. Advances in Physiology Education, v.26, n.4, p.238-255, 2002. Disponível em: <http:// advan.physiology.org/content/26/4/238.full.pdf $>$. Acesso em: 19 mar. 2013. doi: 10.1152/ advan.00039.2002.

IMAIZUMI, T. et al. The role of voltage-gated $\mathrm{Ca}^{+2}$ channels in anoxic injury of spinal cord matter. Brain Research, v.817, p.84-92, 1999. Disponível em: <http://dx.doi.org/10.1016/S00068993(98)01214-1>. Acesso em: 19 mar. 2013. doi: 10.1016/S00068993(98)01214-1.

KAPOOR, V.K. Natural toxins and their therapeutic potential. Indian Journal of Experimental Biology, v.48, p.228-237, 2010. Disponível em: <http://nopr.niscair.res.in/ bitstream/123456789/7397/ 1/IJEB\%2048(3)\%20228-237.pdf>. Acesso em: 19 mar. 2013. PMID: 21046975.

KWON, B.K. et al. Pathophysiology and pharmacologic treatment of acute spinal cord injury. Spine Journal, v.4, p.451-464, 2004. Disponível em: <http://dx.doi.org/10.1016/j.spinee.2003.07.007>. Acesso em: 11 mar. 2013. doi: 10.1016/j.spinee.2003.07.007.

LAVOR, M.S.L. Efeito do etomidato na isquemia e no trauma medular agudo em ratos. 2013. 146p. Tese (Doutorado em Ciência Animal) - Escola de Veterinária, Universidade Federal de Minas Gerais, Belo Horizonte.

LEE, B.B. et al. The global map for traumatic spinal cord injury epidemiology: update 2011, global incidence rate. Spinal Cord, p. 1-7, 2013. Disponível em: <http://dx.doi.org/10.1038/ sc.2012.158>. Acesso em: 11 mar. 2013. doi: 10.1038/sc.2012.158

LEPORE, A.C. et al. Spatial and Temporal Changes in Promoter Activity of the Astrocyte Glutamate Transporter GLT1 Following Traumatic Spinal Cord Injury. Journal of Neuroscience Research, v. 89, p. 1001-1017, 2011. Disponível em: <http:// dx.doi.org/10.1002/jnr.22624>. Acesso em: 11 mar. 2013. doi: 10.1002/jnr.22624.

LEWIS, R.J.; GARCIA, M.L. Therapeutic potencial of venom peptides. Nature Reviews, v.2, p.790-802, 2003. Disponível em: $<$ http://dx.doi.org/10.1038/nrd1197>. Acesso em: 11 mar. 2013. doi: $10.1038 /$ nrd1197.

LIU, H. et al. Identification of three subunits of the high affinity $\omega$-conotoxin MVIIC-sensitive $\mathrm{Ca}^{2+}$ channel. Journal of Biological Chemistry, v.271, n.23, p.13804-13810, 1996. Disponível em: $<$ http://www.jbc.org/content/271/23/13804>. Acesso em: $11 \mathrm{mar}$. 2013. doi: $10.1074 /$ jbc.271.23.13804

LIVETT, B.G. et al. Drugs from the sea: conopeptides as potential therapeutics. Current Medicinal Chemistry, v.11, p.1715-1723, 2004. Disponível em: <http://dx.doi. org/10.2174/0929867043364928 >. Acesso em: 11 mar. 2013. doi: $10.2174 / 0929867043364928$.

MALMBERG, A.B. et al. Powerful antinociceptive effects of the cone snail venom-derived subtype-selective NMDA-receptor antagonists conantokins G and T. Pain, v.101, p.109-116, 2003. Disponível em: $<$ http://dx.doi.org/10.1016/S0304-3959(02)00303-2>. Acesso em: 11 mar. 2013. doi: 10.1016/ S0304-3959(02)00303-2.

MALMBERG, A.B.; YAKSH, T.L. Voltage-sensitive calcium channels in spinal nociceptive processing: blockade of $\mathrm{N}$ - and P-type channels inhibits formalin-induced nociception. Journal of Neuroscience, v.14, n.8, p.4882-4890, 1994. Disponível em: $<\mathrm{http} / / /$ www.jneurosci.org/content/ 14/8/4882>. Acesso em: 11 mar. 2013. PMID: 8046458.

MCDONOUGH, S.I. et al. Interactions among toxins that inhibit N-type and P-type calcium channels. Journal of General Physiology, v.119, p.313-328, 2002. Disponível em: <http:// dx.doi.org/10.1085/ jgp.20028560>. Acesso em: 11 mar. 2013. doi: $10.1085 /$ jgp. 20028560.

MEHDIRATTA, R.; SABERWAL, G. Bio-business in brief: the case of conotoxins. Current Science, v.92, p.39-45, 2007. Disponível em: <http://www.iisc.ernet.in/currsci/jan102007/39. pdf $>$. Acesso em: 11 mar. 2013.

Ciência Rural, v.44, n.3, mar, 2014. 
MENDES, D.S.; ARIAS, M.V.B. Traumatismo da medula espinhal em cães e gatos: estudo retrospectivo de 57 casos. Pesquisa Veterinária Brasileira, v.32, n.12, p.1304-1312, 2012. Disponível em: <http://dx.doi.org/10.1590/S0100736X2012001200015>. Acesso em: 11 mar. 2013. doi: 10.1590/ S0100-736X2012001200015

MINAMI, K. et al. Role of $\mathrm{Thr}^{11}$ in the binding of $\omega$-conotoxin MVIIC to N-type $\mathrm{Ca}^{2+}$ channels. FEBS Letters, v.491, p.127130, 2001. Disponível em: <http://dx.doi.org/10.1016/S00145793(01)02183-4>. Acesso em: 11 mar. 2013. doi: 10.1016/S00145793(01)02183-4

NEBE, J. et al. Spinal application of $\omega$-conotoxin GIVA, an $\mathrm{N}$-type calcium channel antagonist, attenuates enhancement of dorsal spinal neuronal responses caused by intra-articular injection of mustard oil in the rat. Experimental Brain Research, v.120, p.61-69, 1998. Disponível em: <http://link.springer.com/ article/10.1007/s002210050378>. Acesso em: 11 mar. 2013. doi: $10.1007 / \mathrm{s} 002210050378$.

OLBY, N. et al. Long-term functional outcome of dogs with severe injuries of the thoracolumbar spinal cord: 87 cases (1996-2001). Journal of the American Veterinary Medical Association, v.222, n.6, p.762-769, 2003. Disponível em: <http://dx.doi.org/10.2460/ javma.2003.222.762>. Acesso em: 11 mar. 2013. doi: 10.2460/ javma.2003.222.762.

OLIVEIRA, K.M. Efeitos de diferentes doses de $\omega$-conotoxina MVIIC no tratamento de ratos submetidos ao trauma medular agudo compressivo. 2012. 61f. Dissertação (Mestrado em Medicina e Cirurgia Veterinárias) - Escola de Veterinária, Universidade Federal de Minas Gerais, Belo Horizonte, MG.

OLIVERA, B.M. et al. Calcium channel diversity and neurotransmitter release: the $\omega$-conotoxins and $\omega$-agatoxins. Annual Review of Biochemistry, v.63, p.823-867, 1994. Disponível em: $<$ http://dx.doi.org/10.1146/annurev.bi.63.070194.004135>. Acesso em: 11 mar. 2013. doi: 10.1146/annurev.bi.63.070194.004135.

OLIVERA, B.M. et al. Neuronal calcium channel antagonists. Discrimination between calcium channel subtypes using $\omega$-conotoxin from Conus magus venom. Biochemistry, v.26, p.2086-2090, 1987. Disponível em: <http://dx.doi.org/10.1021/ bi00382a004>. Acesso em: 11 mar. 2013. doi: 10.1021/ bi00382a004.

OLIVERA, B.M. et al. Purification and sequence of presynaptic peptide toxin from Conus geographus venom. Biochemistry, v.23, p.5087-5090, 1984. Disponível em: <http://dx.doi.org/ 10.1021/bi00317a001>. Acesso em: 11 mar. 2013. doi: 10.1021/ bi00317a001.

OLIVERA, B.M.; CRUZ, L.J. Conotoxins, in retrospect. Toxicon, v.39, p.7-14, 2001. Disponível em: Acesso em: 11 mar. 2013. Disponível em: <http://dx.doi.org/10.1016/S0041-0101(00)001574>. Acesso em: 11 mar. 2013. doi: 10.1016/S0041-0101(00)00157-4.

OLIVERA, B.M.; TEICHERT, R.W. Diversity of the neurotoxic Conus peptides: a model of concerted pharmacological discovery. Molecular Interventions, v.7, n.5, p.251-260, 2007. Disponível em: <http://dx.doi.org/10.1124/mi.7.5.7>. Acesso em: 11 mar. 2013. doi: $10.1124 / \mathrm{mi} .7 .5 .7$.

OYINBO, C.A. Secondary injury mechanisms in traumatic spinal cord injury: a nugget of this multiply cascade. Acta Neurobiologiae
Experimentalis, v. 71, p. 281-299, 2011. Disponível em: <http:// www.ncbi.nlm.nih.gov/pubmed/21731081\#>. Acesso em: 11 mar. 2013. PMID: 21731081.

PINHEIRO, A.C.N. et al. Phoneutria spider toxins block ischemia-induced glutamate release, neuronal death, and loss of neurotransmission in hippocampus. Hipoccamppus, v.19, p.1123-1129, 2009. Disponível em: <http://dx.doi.org/10.1002/ hipo.20580>. Acesso em: 11 mar. 2013. doi: 10.1002/ hipo.20580.

ROBICHAUD, L.J. et al. The voltage-sensitive $\mathrm{Ca}^{2+}$ channel (VSCC) antagonists omega-Aga-IVA and omega-CTX MVIIC inhibits spontaneuousepileptiform discharges in the rat cortical wedge. BrainResearch, v.643, p.352-356, 1994. Disponível em: $<$ http://dx.doi.org/10.1016/0006-8993(94)90047-7>. Acesso em: 11 mar. 2013. doi: 10.1016/0006-8993(94)90047-7.

ROSADO, I.R. Efeitos do dantrolene sódico e succinato de metilprednisolona na neuroproteção e morte celular na medula espinhal de ratos submetidos a trauma medular compressivo agudo. 2011. 45f. Dissertação (Mestrado em Ciência Animal) - Escola de Veterinária, Universidade Federal de Minas Gerais, MG.

ROSSIGNOL, S. et al. Spinal cord injury: time to move? Journal of Neuroscience, v.27, n.44, p.11782-11792, 2007. Disponível em: $<$ http://dx.doi.org/10.1523/JNEUROSCI.3444-07.2007>. Acesso em: 11 mar. 2013. doi: 10.1523/JNEUROSCI.3444-07.2007.

ROWLAND, J.W. et al. Current status of acute spinal cord injury pathophysiology and emerging therapies: promise on the horizon. Neurosurgery Focus, v.25, n.5, suppl.E2, p.1-17, 2008. Disponível em: <http://dx.doi.org/10.3171/FOC.2008.25.11.E2>. Acesso em: 11 mar. 2013. doi: 10.3171/ FOC.2008.25.11.E2.

SCHWARTZ, G.; FEHLINGS, M.G. Evaluation of the neuroprotective effects of sodium channel blockers after spinal cord injury: improved behavioral and neuroanatomical recovery with riluzole. Journal of Neurosurgery, v.94, p.245-256, 2001. Disponível em: <http://dx.doi.org/10.3171/ spi.2001.94.2.0245>. Acesso em: 11 mar. 2013. doi: 10.3171/ spi.2001.94.2.0245.

SEVERO, M.S. et al. Fisiopatologia do trauma e da compressão à medula espinal de cães e gatos. Medicina Veterinária, v.1, n.2, p.78-85, 2007. Disponível em: <http://www.revista.dmv.ufrpe.br/ index.php/rdmv/article/view/41/41>. Acesso em: 11 mar. 2013.

SHEN, G.S. et al. Conopeptides: from deadly venoms to novel therapeutics. DDT, v.5, n.3, p.98-105, 2000. Disponível em: $<$ http://dx.doi.org/10.1016/S1359-6446(99)01454-3>. Acesso em: 11 mar. 2013. doi: 10.1016/S1359-6446(99)01454-3.

SULLIVAN, P.G. et al. Mitochondrial uncoupling as a therapeutic target following neuronal injury. Journal of Bioenergetics and Biomembranes, v.36, n.4, p.353-356, 2004. Disponível em: <http:// dx.doi.org/10.1023/B:JOBB.0000041767.30992.19>. Acesso em: 11 mar. 2013. doi: 10.1023/ B:JOBB.0000041767.30992.19.

VALENTINO, K. et al. A selective N-type calcium channel antagonist protects against neuronal loss after global cerebral ischemia. Proceeding of the National Academy of Sciences of the United States, v.90, p.7894-7897, 1993. Disponível em: $<$ http://dx.doi.org/10.1073/pnas.90.16.7894>. Acesso em: 11 mar. 2013. doi: 10.1073/pnas.90.16.7894.

Ciência Rural, v.44, n.3, mar, 2014. 
VERWEIJ, B.H. et al. Mitochondrial dysfunction after experimental and human brain injury and its possible reversal with a selective N-type calcium channel antagonist (SNZ-111). Neurol. Res., v. 19, p. 334-339, 1997. Disponível em: < http://dx.doi.org/10.1016/ S0303-8467(97)81724-6>. Acesso em: 11 mar. 2013. doi: 10.1016/ S0303-8467(97)81724-6.

WATTERS, M.R.; STOMMEL, E.W. Marine neurotoxins: envenomations and contact toxins. Current Treatment Options in Neurology, v.6, p.115-123, 2004. Disponível em: < http://dx.doi. org/ 10.1007/s11940-004-0021-8>. Acesso em: 11 mar. 2013. doi: 10.1007/s11940-004-0021-8.

WU, Y. et al. Delayed post-injury administration of riluzole is neuroprotective in a preclinical rodent model of cervical spinal cord injury. Journal of Neurotrauma, v.30, p.1-12, 2013. Disponível em: <http://dx.doi.org/10.1089/neu.2012.2622>. Acesso em: 11 mar. 2013. doi: 10.1089/neu.2012.2622.

YAMADA, K. et al. $\omega$-conotoxin GIVA protects against ischemia-induced neuronal death in the Mongolian gerbil but not against quinolinic acid-induced neurotoxicity in the rat. Neuropharmacology, v.33, n.2, p.251-254, 1994. Disponível em: $<$ http://dx.doi.org/10.1016/0028-3908(94)90016-7>. Acesso em: 11 mar. 2013. doi: 10.1016/0028-3908(94)90016-7.

ZARDO, K.M. et al. Contribuição das projeções oblíquas em mielografias de pequenos animais para localização de lesões medulares causadas por processo degenerativo intervertebral. Ciência Rural, v.40, n.11, p.2324-2331, 2010. Disponível em: $<$ http://dx.doi.org/10.1590/S0103-84782010001100012>. Acesso em: 11 mar. 2013. doi: 10.1590/S0103-84782010001100012. 